\title{
Organization of Radar Survey \\ in a Multi-Position Radar System \\ With Non-Operated Illumination Source Based \\ on Combinatorial Principle
}

\author{
Denis M. Petrochenkov and Aleksandr V. Fedotov* \\ "Yaroslavl Higher Military Institute of the Air Defense" \\ of the Ministry of Defense of the Russian Federation \\ 28 Moscow, Yaroslavl, 150001, Russia
}

Received 26.07.2018, received in revised form 14.09.2018, accepted 27.10.2018

\begin{abstract}
A version of designing multiple semiactive radar system (radar), consisting of receiving stations with multi-beam directional diagrams of the antennas, performing the review space and detection of aerial objects on the basis of the combinatory principles. The evaluation of the potential of the semi-active radar on spatial indicators. Proposed criteria to develop recommendations to build spatial configuration of multi-position radar system.
\end{abstract}

Keyword: semi-active multi-position radar system, multi-beam directional diagram, non-search method of review.

Citation: Petrochenkov D.M., Fedotov A.V. Organization of radar survey in a multi-position radar system with non-operated illumination source based on combinatorial principle, J. Sib. Fed. Univ. Eng. technol., 2018, 11(7), 831-841. DOI: 10.17516/1999494X-0098.

(C) Siberian Federal University. All rights reserved

This work is licensed under a Creative Commons Attribution-NonCommercial 4.0 International License (CC BY-NC 4.0).

* Corresponding author E-mail address: pdm78@mail.ru, alekcandr1384@yandex.ru 


\title{
Организация обзора пространства
}

\section{В многопозиционной радиолокационной системе \\ с некооперируемым источником подсвета \\ на основе комбинаторного принципа}

\author{
Д.М. Петроченков, А.В. Федотов \\ Ярославское высшее военное училище \\ противовоздушной оборонь \\ Министерство обороны Российской Федерачии
} Россия, 150001, Ярославль, Московский проспект, 28

В статье представлен вариант построения полуактивной многопозиционной радиолокационной системы (МПРЛС), состоящей из приемных пунктов с многолучевыми диаграммами направленности антенн, осуществляющих обзор пространства и обнаружение воздушных объектов на основе комбинаторных принципов. Проведена оценка потенциальных возможностей полуактивной МПРЛС по пространственным показателям. Предложены критерии, позволяющче выработать рекомендации к построению пространственной конфигураџии МПРЛС.

Ключевые слова: полуактивная многопозиционная радиолокационная система, многолучевая диаграмма направленности, беспоисковый способ обзора.

Многопозиционная радиолокационная система (МПРЛС) состоит из отдельных радиолокационных станций (РЛС), являющихся ее подсистемами, которые могут быть активными, полуактивными, пассивными. В случае полуактивной многопозиционной радиолокационной системы в качестве источника подсвета могут выступать кооперируемые (собственные) и некооперируемые источники подсвета (НИП) - источники противоборствующей стороны в конфликте или нейтральной стороны. Качество системы определяется не только количественными характеристиками отдельно взятых элементов (подсистем), но и количественными характеристиками связей подсистем друг с другом.

Исследованию МПРЛС со сторонними источниками подсвета (кооперируемыми и некооперируемыми) посвящено большое количество работ как отечественных, так и зарубежных авторов. В работах [1-6] МПРЛС рассматривается с различных сторон, оценивается различными показателями, однако вопросы оценки количественных характеристик связей между ее элементами (подсистемами) освещаются недостаточно.

На современном этапе развития технологий информационного обмена можно обеспечить достаточно большой по протяженности и пропускной способности канал передачи информации, но в ряде случаев даже современные технологии не могут обеспечить его высокое качество. К таким случаям можно отнести:

- современные РЛС и МПРЛС должны быть мобильными, тогда при смене позиций могут возникать различные условия, не позволяющие обеспечить высокое качество канала информационного обмена (рельеф, застройка, электромагнитная совместимость с соседними радиоэлектронными средствами (РЭС) и т.д.); 
- при использовании МПРЛС в условиях антагонистического конфликта обеспечение высокого качества канала информационного обмена будет затруднено из-за воздействий противостоящей стороны (уничтожение, повреждение, радиоэлектронное подавление и т.д.);

- при построении МПРЛС с НИП обеспечение качества за счет повышения требований к количественным характеристикам канала информационного обмена повышает уязвимость системы, отказ канала информационного обмена приведет к отказу всей системы.

Таким образом, по мнению авторов, основной принцип организации информационного обмена в МПРЛС с НИП можно сформулировать так: зависимость качества МПРЛС с НИП от количественных характеристик канала информационного обмена должна быть минимальна.

Данный принцип противоречив, решение противоречия может быть одним из подходов к развитию МПРЛС с НИП.

В настоящей работе предметом рассмотрения является возможная реализация обзора пространства МПРЛС с НИП, позволяющая снизить требования к количественным характеристикам канала информационного обмена за счет уменьшения объема передаваемой информации.

В пассивной радиолокации обзор пространства возможно реализовать поисковым и беспоисковым способами.

При реализации поискового способа (рис. 1) обзор пространства обеспечивается электронным, механическим или комбинированным способом качания луча диаграммы направленности антенной системы (ДНА). При этом возникают ограничения на возможности обзора: необходимо решать задачи выбора периода обзора и синхронизации обзора пространства приемными пунктами, что достаточно трудно реализовать.

Беспоисковый способ пеленгации реализуется применением всенаправленных антенн, антенных устройств с многолучевыми статичными диаграммами направленности (рис. 2).

Рассмотрим МПРЛС, состоящую из трех приемных пунктов (ПП), с многолепестковыми (многолучевыми) ДН антенн в азимутальной плоскости (рис. 2). Каждый ПП МПРЛС, изображенный на рис. 2, имеет ДНА, состоящую из 32 лепестков с шириной каждого лепестка приблизительно 5,6², данные значения выбраны в качестве примера.

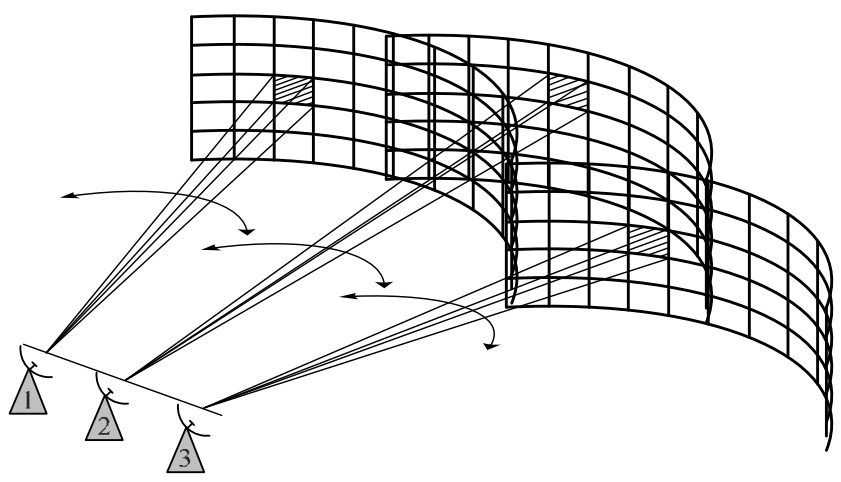

Рис. 1. Поисковый способ обзора пространства МПРЛС

Fig. 1. Search method of the survey space multi-radar system 


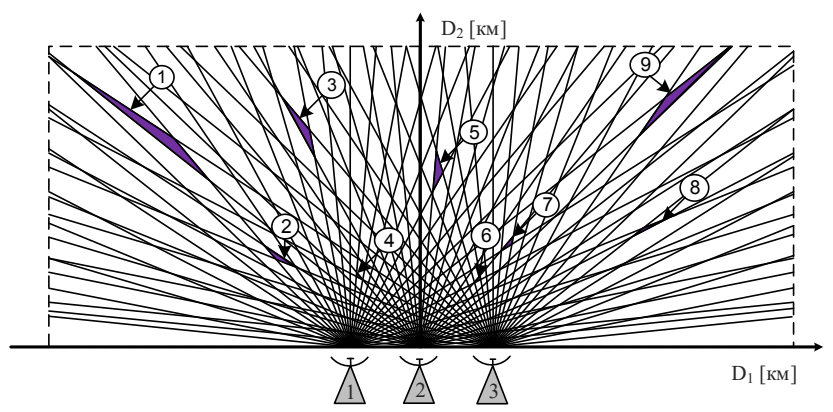

Рис. 2. Беспоисковый способ обзора МПРЛС с многолучевыми ДНА ПП

Fig. 2. A searchless way to review a multi-position radar system with multipath antenna patterns of receiving points

Если каждому лепестку ДН ПП присвоить номер от 1 до $N$ (где $N$ - число лепестков ДНА) и разместить ПП на некотором удалении друг от друга, то каждой точке оцениваемого пространства можно поставить в соответствие комбинацию чисел - номеров лепестков ДН ПП, то есть сформировать своего рода систему координат.

Например, на рис. 2 представлены 9 закрашенных областей пространства, выбранных случайным образом и обозначенных цифрами. Данные области получаются следующим образом: если многолепестковые ДНА 1-го, 2-го и 3-го ПП принять соответственно за генеральные совокупности множеств статичных секторов обзора ПП (1):

$$
\begin{aligned}
& A=\left\{a_{1}, a_{2}, a_{3}, \ldots, a_{n}\right\}, n=\left(\beta / \beta_{л}+1\right)-\text { челое, } \\
& B=\left\{b_{1}, b_{2}, a_{3}, \ldots, b_{n}\right\}, n=\left(\beta / \beta_{л}+1\right)-\text { целое }, \\
& C=\left\{c_{1}, c_{2}, c_{3}, \ldots, c_{n}\right\}, n=\left(\beta / \beta_{л}+1\right)-\text { целое },
\end{aligned}
$$

где $A$ - множество статичных секторов обзора ПП А; $\beta$ - сектор обзора ПП; $\beta$ - ширина одного лепестка ДНА; $n$ - количество лепестков ДНА ПП; $B$ - множество статичных секторов обзора ПП В; $C$ - множество статичных секторов обзора ПП С, то взаимные пересечения лепестков ДНА ПП образуют области пространства, которые будем называть объемами взаимного разрешения. Множество объемов взаимного разрешения обозначим

$$
X=\left(x_{1}, x_{2}, x_{3}, \ldots, x_{k}\right), k \text {-иелое }
$$

Множество $X$ получается на основе пересечений множеств $A, B$ и $C, M$ - число возможных пересечений ДНА всех ПП (мощность множества $X$ ).

$$
\begin{aligned}
& X=A \cap B \cap C \Leftrightarrow x_{i} \in a_{k}, a_{k} \in A, k \in\{1,2,3, \ldots, N\} \wedge \\
& \wedge x_{i} \in b_{s}, b_{s} \in B, s \in\{1,2,3, \ldots, N\} \wedge \\
& \wedge x_{i} \in c_{u}, c_{u} \in C, u \in\{1,2,3, \ldots, N\} .
\end{aligned}
$$

Выбранные области принадлежат множеству $X$.

Если обозначить номера лепестков каждого из трех ПП справа налево (рис. 3), то $x_{\mathrm{k}}, k \in\{1, \ldots, 9\}$ (закрашенные области) соответствуют комбинации чисел, представленных в табл. 
Таблица

Table

\begin{tabular}{|c|c|c|c|c|c|c|c|c|c|}
\hline \multirow{2}{*}{ Номер ПП } & \multicolumn{9}{|c|}{ Номер оцениваемой области } \\
\cline { 2 - 12 } & 1 & 2 & 3 & 4 & 5 & 6 & 7 & 8 & 9 \\
\hline 1 & 9 & 10 & 15 & 19 & 22 & 28 & 27 & 28 & 27 \\
\hline 2 & 8 & 6 & 23 & 9 & 18 & 24 & 23 & 27 & 23 \\
\hline 3 & 6 & 5 & 9 & 6 & 13 & 14 & 18 & 26 & 23 \\
\hline
\end{tabular}

Таким образом, если в одной из областей пространства, характеризуемой множеством $x_{i}$, будет воздушный объект, то определение его местоположения возможно по анализу в пункте совместной обработки информации комбинации номеров каналов ПП, в которых осуществлен прием сигнала от воздушного объекта.

За счет передачи между приемными пунктами и пунктом совместной обработки только служебной информации и номеров каналов, в которых получены сигналы, снижаются требования к количественным характеристикам каналов информационного обмена. По комбинации каналов можно отождествить воздушный объект и однозначно определять положение воздушных объектов в пространстве.

Число возможных комбинаций каналов ПП, без учета пространственного расположения, составит:

$$
m=n^{Q},
$$

где Q - число ПП в МПРЛС; $n$ - число лепестков в ДНА каждого ПП (при условии, что ДНА ПП одинаковы).

Для рассматриваемого примера число возможных комбинаций - размещений, исходя из теории комбинаторики формулы (4), составит $m=32768$, но такого числа взаимных пересечений лепестков ДНА не будет за счет пространственного разноса ПП и ДНА в пространстве. Например, 1-й лепесток первого ПП не имеет взаимных пересечений с 32-м лепестком третьего ПП.

Проведем оценку комбинаторного подхода в организации обзора пространства в МПРЛС, в качестве количественных показателей выберем:

- число объемов взаимного разрешения, мощность множества: $|X|=M$;

- максимальную площадь сечения объемов взаимного разрешения ДНА: $\max (|x i|)$,

$\begin{aligned} & \mathrm{i} \in\{1,2,3, \ldots, M\} ; \\ \text { - } & \text { среднюю величину площади сечения объемов взаимного разрешения ДНА: } \frac{\sum_{i=1}^{M}\left|x_{i}\right|}{M} \text {. }\end{aligned}$

В качестве аргументов при оценке указанных выше показателей выберем: ширину одного лепестка ДНА ПП по азимуту ( $)$ и удаление одного из ПП от центрального $\left(\mathrm{R}_{13}-\right.$ для системы из трех и $\mathrm{R}_{14}$ - для системы из четырех ПП).

На рис. 4 приводится результат оценки мощности множества $|X|$ в зависимости от выбранных аргументов: ширина луча диаграммы ДНА: $5^{\circ}, 10^{\circ}, 15^{\circ}, 20^{\circ}$, удаление ПП от центрального: 


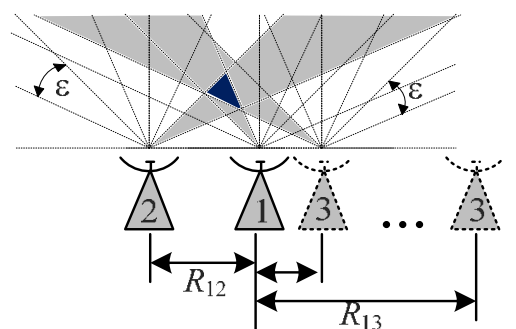

a

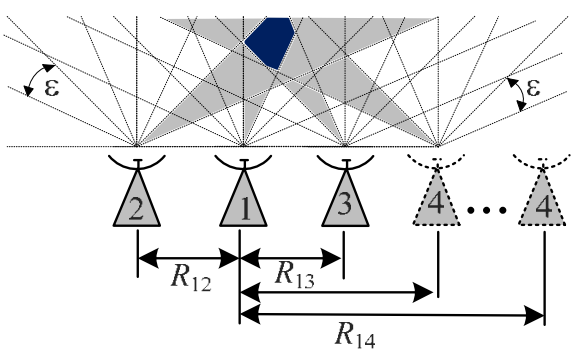

б

Рис. 3. Методика оценки показателей МПРЛС при беспоисковом способе обзора: a - из трех ПП (R $\mathrm{R}_{13}-$ аргумент пространственной конфигурации системы); б-из четырех ПП ( $\mathrm{R}_{14}$-аргумент пространственной конфигурации системы)

Fig. 3. Methodology for estimating the performance of a multi-position radar system with a searchless survey method: $a-$ of 3 receiving points $\left(R_{13}\right.$ is the argument of spatial configuration of the system); $\sigma$ - of 4 receiving points $\left(\mathrm{R}_{14}\right.$ is the argument of spatial configuration of the system)

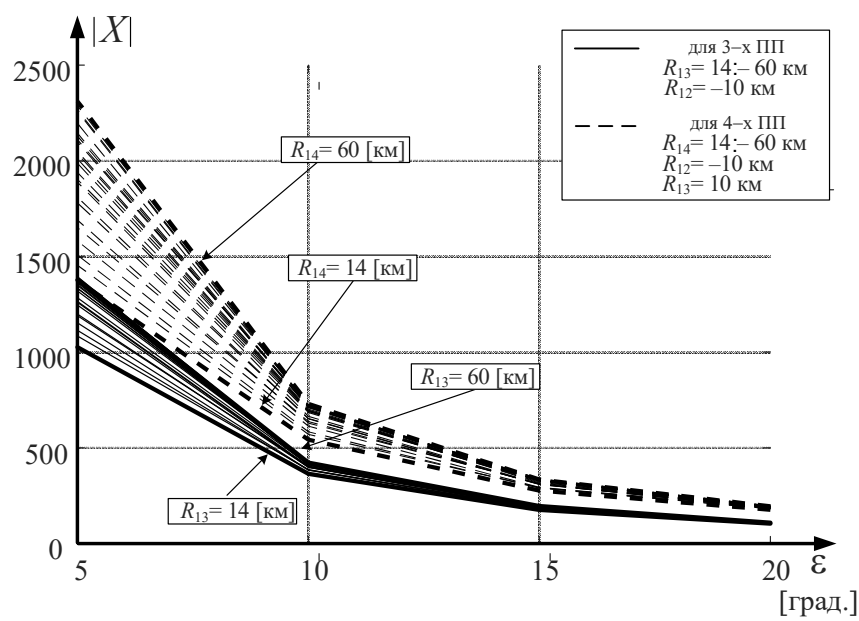

Рис. 4. Кривые оценки мощности множества объемов взаимного разрешения от ширины луча ДНА ПП и аргументов пространственной конфигурации МПРЛС $\left(\mathrm{R}_{13}, \mathrm{R}_{14}\right)$

Fig. 4. Curves for estimating the power of a set of volumes of mutual resolution of the beam width of the antenna of receiving points and spatial configuration arguments $\left(\mathrm{R}_{13}, \mathrm{R}_{14}\right)$

- системы из трех ПП (рис. $3 a$ ) $\mathrm{R}_{13}=14 \div 60$ км с шагом в 2 км, при $\mathrm{R}_{12}=10$ км;

- системы из четырех ПП (рис. 36 ) $\mathrm{R}_{14}=14 \div 60$ км с шагом в 2 км, $\mathrm{R}_{12}=10$ км, $\mathrm{R}_{13}=-10$ км;

- оцениваемая область пространства имеет размеры (рис. 2): $\mathrm{D}_{1}=-300 \div 300$ км, $\mathrm{D}_{2}=0 \div 300$ км.

Полученные результаты позволяют сделать следующие выводы:

- зависимость $|X|\left(\varepsilon, R_{1 x}\right)$ носит нелинейный характер;

- для случаев трех и четырех позиций ПП зависимости $|X|\left(\varepsilon, R_{13}\right)$ и $|X|\left(\varepsilon, R_{14}\right)$ имеют одинаковую закономерность. За счет увеличения числа ПП и уменьшения ширины лепестков ДНА происходит увеличение $|X|$. При этом большее влияние оказывает ширина лепестка ДНА ( $)$; 
- при значении ширины лепестка ДНА $\varepsilon>15^{\circ}$ число ПП, их взаимное расположение не оказывают существенного влияния на $|X|$ и, как следствие, на точность таких систем;

- при ширине лепестка ДНА $\varepsilon<10^{\circ}$ число ПП, их взаимное положение существенно влияют на $|X|$;

- при малых значениях ширины ДНА за счет оптимального размещения ПП можно добиться увеличения $|X|$.

Следует отметить, что системой из трех ПП можно добиться такого же показателя $|X|$, как и для системы из четырех ПП, за счет оптимального размещения ПП.

На рис. 5 представлен результат оценки максимальной площади сечения объема взаимного разрешения $\max \left(\left|x_{i}\right|\left(\varepsilon, R_{1 x}\right)\right), i \in\{1,2,3, \ldots, M\}$, в зависимости от ширины лепестка ДНА в азимутальной плоскости при перемещении одного из ПП относительно центрального при $\mathrm{D}_{1}=-300 \div 300$ км, $\mathrm{D}_{2}=0 \div 300$ км: a) - система из трех ПП, б) - система из четырех ПП. Полученный результат представлен в нормированном, прологарифмированном виде по значению максимальной площади сечения объема взаимного разрешения Хтах при ширине лепестка ДНА ПП $\varepsilon=20^{\circ}$, для удобства визуального представления и оценки малых диапазонов изменения исследуемых зависимостей.

Полученные зависимости на рис. 5 позволяют сделать выводы:

- для системы из трех ПП их расположение оказывает влияние на значение $\max \left(\left|x_{i}\right|\left(\varepsilon, R_{13}\right)\right)$. Явно это проявляется при малых значениях ширины лепестков ДНА: так, при $\varepsilon=5^{\circ}$ увеличение наблюдается в диапазоне $R_{13}=25 \div 38$ км. При $\varepsilon=10^{\circ}$ увеличение начинается с $R_{13}=45 \kappa м$. При значениях $\varepsilon=15^{\circ}$ и $\varepsilon=20^{\circ}$ отмеченного увеличения нет, так как оно не попадает в оцениваемый диапазон дальностей $\mathrm{D}_{1}$ и $\mathrm{D}_{2}$ (рис. 2);

- для системы из трех ПП на всех четырех зависимостях можно отметить оптимальное (наименьшее) расстояние удаления третьего ПП от центрального. Так, при $\varepsilon=5^{\circ}$ удале-

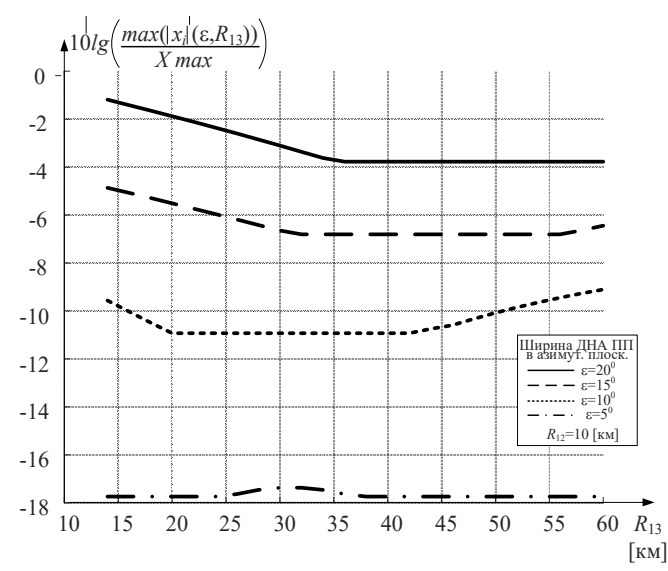

a)

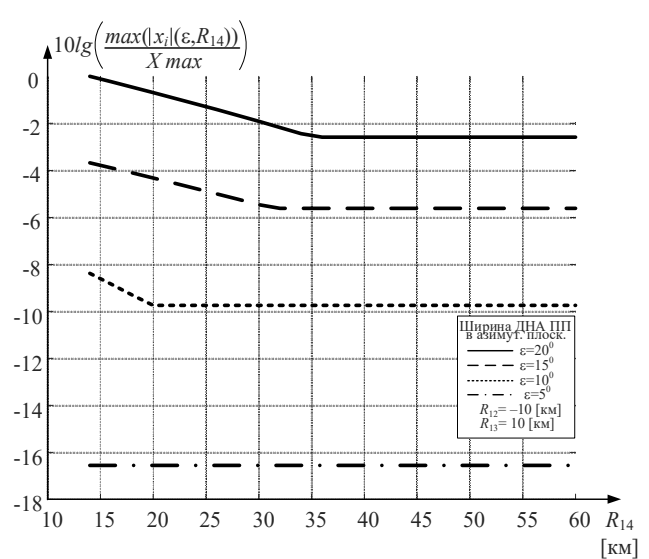

б)

Рис. 5. Оценка максимальной площади сечения объемов взаимного разрешения МПРЛС для оцениваемого пространства $\mathrm{D}=600$ *300 км: a - МПРЛС из трех ПП; б - МПРЛС из четырех ПП

Fig. 5. Estimation of the maximum cross-sectional area of volumes of mutual resolution of a multi-position radar system for the estimated space $\mathrm{D}=600 * 300 \mathrm{~km}$ : a - from 3 receiving points; $\sigma-$ from 4 receiving points 
ние составляет $R_{13}=10 \mathrm{\kappa m}$, при $\varepsilon=10^{\circ} R_{13}=20 \mathrm{\kappa m}$, при $\varepsilon=15^{\circ} R_{13}=33 \mathrm{\kappa m}$, при $\varepsilon=20^{\circ} R_{13}=35 \mathrm{\kappa m}$. Можно утверждать, что с дальнейшим увеличением ширины лепестков ДНА приращения уменьшаются;

- для системы из четырех ПП зависимость $\max \left(\left|x_{i}\right|\left(\varepsilon, R_{14}\right)\right)$ уменьшается. При малой ширине лепестков ДНА $\left(\varepsilon=5^{\circ}\right)$ зависимость в оцениваемом диапазоне дальностей отсутствует. При больших значениях ширины лепестков ДНА $\left(\varepsilon=10^{\circ} \div 20^{\circ}\right)$ можно отметить оптимальные, минимальные удаления, причем они совпадают с показателями, указанными выше для системы из трех ПП.

Однако полученные результаты были рассчитаны для оцениваемого пространства размерами 600 км на 300 км. Если провести оценку МПРЛС по тому же критерию, но для меньшего оцениваемого пространства, то получится результат, представленный на рис. 6, 7. На рис. 6 изображен результат оценки $\max \left(\left|x_{i}\right|\left(\varepsilon, R_{1 x}\right)\right), i \in\{1,2,3, \ldots, M\}$ при размерах оцениваемого пространства $\mathrm{D}_{1}=-150 \div 150$ км, $\mathrm{D}_{2}=0 \div 300$ км.

На рис. 7 видим результат оценки $\max \left(\left|x_{i}\right|\left(\varepsilon, R_{13}\right)\right), i \in\{1,2,3, \ldots, M\}$ МПРЛС из трех ПП при размерах оцениваемого пространства $\mathrm{D}_{1}=-75 \div 75$ км, $\mathrm{D}_{2}=0 \div 150$ км.

Полученные зависимости указывают на то, что при оценке необходимо вводить еще один аргумент: $D=\left[D_{1}, D_{2}\right]$, но оптимальное удаление ПП друг от друга выбирать по правилу

$$
R_{1 \text { xопm. }}=\min \left(\max \left(\left|x_{i}\right|\left(\varepsilon, R_{1 x}\right)\right)\right) \text {, при } R_{1 x} \rightarrow 0 .
$$

На рис. 8 представлен результат оценки зависимости средней площади сечения объемов взаимного разрешения МПРЛС из трех и четырех ПП от ширины лепестка ДНА в азимутальной плоскости при перемещении одного из ПП относительно центрального, при $D_{1}=-300 \div 300$ км, $\mathrm{D}_{2}=0 \div 300$ км.

На рис. 9 изображен результат оценки зависимости средней площади сечения объемов взаимного разрешения МПРЛС из трех ПП от ширины лепестка ДНА в азимутальной плоскости

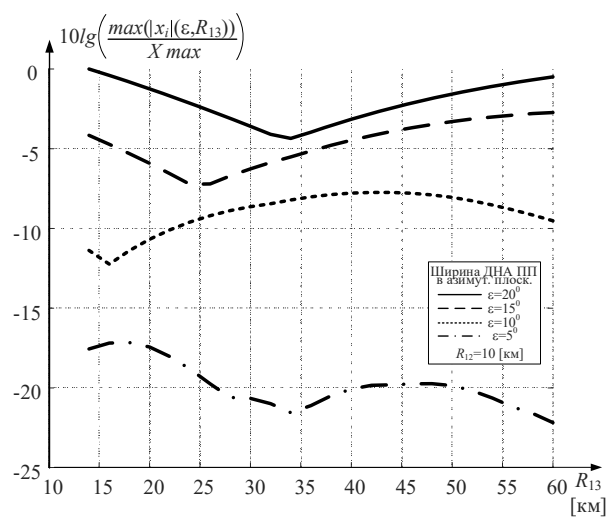

a)

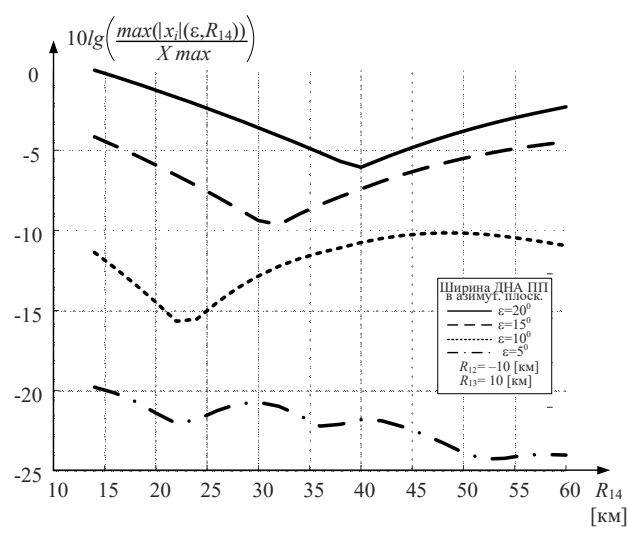

б)

Рис. 6. Оценка максимальной площади сечения объемов взаимного разрешения МПРЛС для оцениваемого пространства $\mathrm{D}=300 * 300$ км: $\mathrm{a}-$ МПРЛС из трех ПП; б - МПРЛС из четырех ПП

Fig. 6. Estimation of the maximum cross-sectional area of volumes of mutual resolution of a multi-position radar system for the estimated space $\mathrm{D}=300 * 300 \mathrm{~km}$ : a - from 3 receiving points; $\sigma-$ from 4 receiving points 


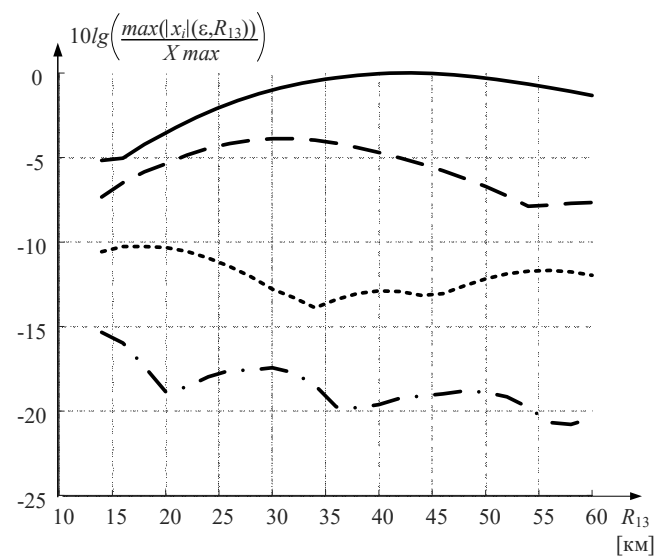

Рис. 7. Оценка максимальной площади сечения объемов взаимного разрешения МПРЛС из трех ПП для оцениваемого пространства $\mathrm{D}=150 * 150$ км

Fig. 7. Estimation of the maximum cross-sectional area of volumes of mutual resolution of a multi-position radar system of 3 receiving points for the estimated space $\mathrm{D}=150 * 150 \mathrm{~km}$

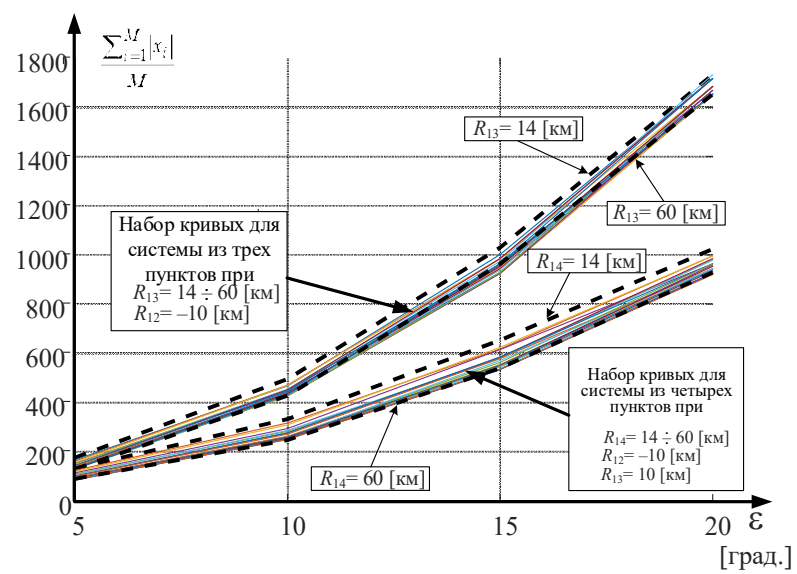

Рис. 8. Кривые средней площади сечения объемов взаимного разрешения МПРЛС

Fig. 8. Curves of the average cross-sectional area volumes of the mutual resolution of the multi-position radar system

при перемещении одного из ПП относительно центрального: а) D1 $=-300 \div 300$ км, D2 $=0 \div 300$ км; б) $\mathrm{D} 1=-150 \div 150$ км, $\mathrm{D} 2=0 \div 300$ км; в) $\mathrm{D} 1=-75 \div 75$ км, $\mathrm{D} 2=0 \div 150$ км.

Анализ полученных результатов показывает:

- изменение аргумента $R_{1 x}$ влияет незначительно на среднюю площадь сечения объема взаимного разрешения. По диапазону семейства кривых можно утверждать, что данная зависимость увеличивается при увеличении числа ПП (семейство кривых для четырех ПП шире, чем семейство кривых для трех ПП);

- изменение аргумента $\varepsilon$ оказывает большее влияние на оцениваемую величину относительно $R_{1 x}$. Величина средней площади сечения объемов взаимного разрешения изменяется в 10 раз при $\varepsilon=20^{\circ}$ относительно величины при $\varepsilon=5^{\circ}$; 


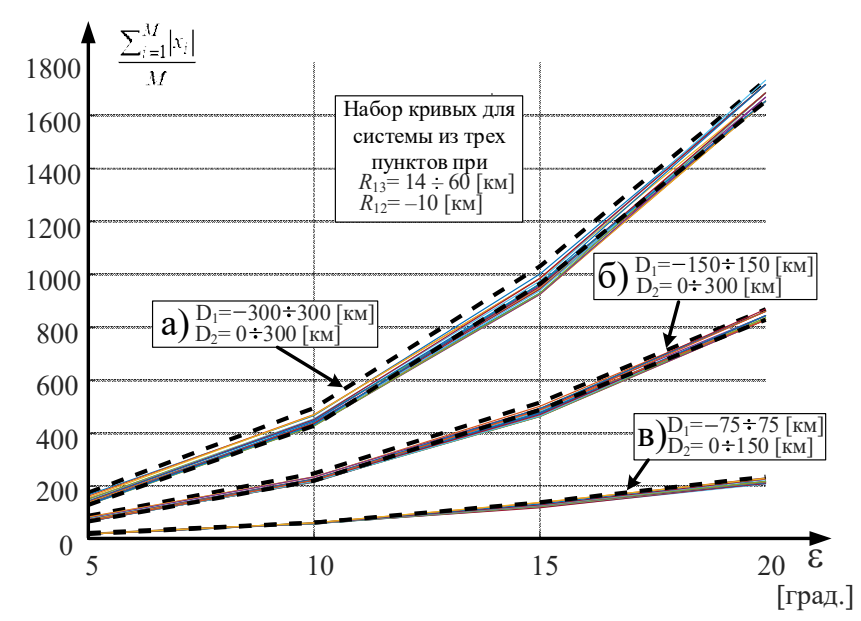

Рис. 9. Кривые средней площади сечения объемов взаимного разрешения МПРЛС

Fig. 9. Curves of the average cross-sectional area of volumes of mutual resolution of a multi-position radar system

- закон изменения средней площади сечений объемов взаимного разрешения для систем из трех и четырех ПП имеет нелинейный характер. Если данную зависимость аппроксимировать параболой

$$
\frac{\sum_{i=1}^{M}\left|x_{i}\right|}{M}(\varepsilon)=a \varepsilon^{2}
$$

то относительно результатов на рис. $9 \alpha_{3 \Pi \Pi}=4,5, \alpha_{4 \Pi \Pi}=2,6$, а отношение $\frac{a_{3 \Pi \Pi}}{a_{4 \Pi \Pi}}=1,7$. Данная пропорция показывает выигрыш системы из четырех ПП относительно системы из трех ПП. Пропорция подтверждается и результатами, полученными при ограничении оцениваемого пространства для значений:

$$
D_{1}=-150 \div 150 \text { км, } D_{2}=0 \div 300 \text { км; } D_{1}=-75 \div 75 \text { км, } D_{2}=0 \div 150 \text { км. }
$$

Таким образом, приведенные выше оценки могут дать минимальные характеристики по точности для решения радиолокационных задач. Если необходимо измерить три координаты $\beta, \varepsilon, r$ воздушного объекта (ВО), то, используя показатели, можно выработать правило:

$$
\max (\Delta \theta, \Delta \beta, \Delta r)=\sup \left(X\left(R_{1 x}, \varepsilon, D\right)\right), X=\left\{x_{1}, x_{2}, x_{3}, \ldots,|X|\right\},
$$

где $\Delta \theta$ - неопределенность в измерении азимута ВО; $\Delta \beta$ - неопределенность в определении угла места ВО; $\Delta r$ - неопределенность в определении дальности ВО.

Исходя из сформулированного правила, можно сделать вывод: ширина луча ДНА должна стремиться к минимуму, оцениваемая область пространства $\left(\mathrm{D}\left[\mathrm{D}_{1}, \mathrm{D}_{2}\right]\right)$ - задаваемая величина (const), $R_{1 x}$ - регулятор пространственной конфигурации МПРЛС - оптимально настраиваемая величина. 


\section{Список литературы}

[1] Ильин Е.М., Климов Н.С., Пащин А.И., Полубехин А.И. и др. Пассивные локационные системы. Перспективы и решения, Вестник СибГУТИ, 2015, (2), 7-20 [Ilyin E.M., Klimov N.S. Pashin A.I., Polybahn A.I. Passive radar systems. Challenges and solutions, Vestnik SibSUTI, 2015, (2), 7-20 (in Russian)].

[2] Онищенко В.С. Пути повышения эффективности систем скрытной радиолокации, Молодой ученый, (12), 353-358 [Onishchenko V.S. Ways to improve the efficiency of covert radar systems, Young scientist, (12), 353-358 (in Russian)].

[3] Батчев С.А., Зайчев А.Г., Талалаев А.Б., Тимаков Д.А. Методы обнаружения и сопровождения воздушных объектов по отраженным радиосигналам сторонних источников в пассивноактивных системах радиолокации, Программные продукты и системы/Software\&Systems, 2016, (3), 168-173 [Batchev S.A., Saichev A.G. Talalaev, A.B., Timakov D.A. Methods for detection and tracking of air objects by reflected radio signals from third-party sources in a passive-active systems, radar systems, Software products and system/Software\&Systems, 2016, (3), 168-173 (in Russian)].

[4] Пархоменко Н.Г., Перетятько А.А., Рейзенкинд Я.А. и др. Применение вариационного метода к задаче оценки параметров сигналов в пассивной радиолокации с посторонним подсветом, Автометрия, 2014, (1), 60-65 [Parkhomenko N.G., Peretyatko A A., Razinkina J.A. etc. the Application of the variational method to the problem of estimating parameters of signals in passive radar with side illumination, Autometry, 2014, (1), 60-65 (in Russian)].

[5] Ашурков И.С., Какаев В.В., Лешко Н.А. Оптимизация пространственной структуры многопозиционной радиолокационной системы, Информационно-управляющие системы, 2015, (6), 81-85 [Ashurkov I.S., Kakaev V.V., Leshko N.A. Optimization of the spatial structure of a multiposition radar system, Information and control systems, 2015, (6), 81-85 (in Russian)].

[6] Бархатов А.В, Веремьев Е.Н., Воробьев А.А. и др. Пассивная когерентная радиолокаиия. СПб.: СПбГЭТУ «ЛЭТИ», 2016 [Barkhatov A.V, Verem’ev E.N., Vorob’ev A.A. and others. The Passive coherent radar. SPb.: Etu "LETI", 2016 (in Russian)]. 